and CD138+ B cells in the SMG tissues and the spleen collected from treated group and control group. (mean $\pm S D ; n=7$ per group; ${ }^{*} \mathrm{P}<0.05$, ${ }^{\star *} \mathrm{P}<0.01$, ${ }^{\star \star *} \mathrm{P}<0.001$ ).

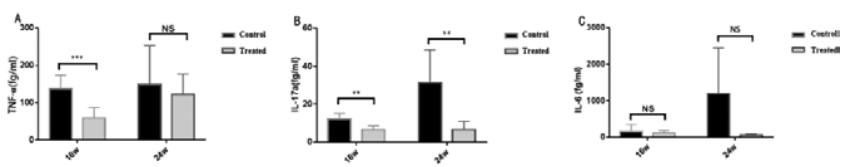

Figure 3. The proinflammatory cytokine levels of TNF-a, IL-17, and IL-6 in the serum samples from the treated and control group. (mean $\pm S D ; n=7$ per group; ${ }^{*} \mathrm{P}<0.05$, ${ }^{* *} \mathrm{P}<0.01$, ${ }^{* * *}$ $\mathrm{P}<0.001)$.

Conclusion: Leflunomide may prevent and improve salivary gland hypofunction and inhibit immune activation in NOD mice, providing a theoretical basis for evaluating leflunomide in the treatment of Sjogren's syndrome.

References:

[1] Van Woerkom JM, Kruize A A, Geenen R, et al. Safety and efficacy of leflunomide in primary Sjögren's syndrome: a phase II pilot study [J]. Ann. Rheum. Dis, 2007, 66(8): 1026-32.

DOI: 10.1136/ard.2006.060905.

Acknowledgments : The authors thank the Center for Scientific Research of Anhui Medical University for valuable help in our experiment.

Disclosure of Interests: : None declared

DOI: 10.1136/annrheumdis-2020-eular.4292

\section{THU0229 HSA-MIR-513C-3P OVEREXPRESSION DECREASES XBP-1S CORRELATING WITH INCREASED INFLAMMATION AND AUTOANTIBODIES IN SALIVARY GLANDS FROM SJÖGREN'S SYNDROME PATIENTS}

P. Carvajal ${ }^{1}$, S. Aguilera ${ }^{2}$, B. Heathcote ${ }^{1}$, I. Castro $^{3}$, D. Jara ${ }^{1}$, M. J. Barrera ${ }^{4}$, V. Maracaja ${ }^{5}$, S. González ${ }^{6}$, V. Aliaga ${ }^{5}$, C. Molina ${ }^{4}$, M. J. González ${ }^{1}$.

${ }^{1}$ Universidad de Chile, Programa de Biología Celular y Molecular, Instituto de Ciencias Biomédicas, Facultad de Medicina, Santiago, Chile; ${ }^{2}$ Clinica Indisa, Reumatología, Santiago, Chile; ${ }^{3}$ Universidad de Chile, Departamento de Tecnología Médica, Facultad de Medicina, Santiago, Chile; ${ }^{4}$ Universidad San Sebastián, Facultad de Odontología, Santiago, Chile; ${ }^{5}$ Universidad de Chile, Departamento de Bioquímica y Biología Molecular, Facultad de Ciencias Químicas y Farmacéuticas, Santiago, Chile; ${ }^{6}$ Universidad Mayor, Escuela de Odontología, Facultad de Ciencias, Santiago, Chile

Background: Endoplasmic reticulum (ER) stress and the Unfolded Protein Response (UPR) are linked to inflammation in a variety of human pathologies including autoimmune diseases. Salivary glands (SG) from Sjögren's syndrome (SS) patients have high levels of IFN-gamma among other cytokines, which trigger or exacerbate protein unfolding or misfolding, inducing ER stress. In this study, we focused on the IRE1a/XBP-1 pathway of the UPR, whose transcription factor is XBP-1s, which induces genes linked to regulation of the secretory pathway. We recently found that SG of SS patients have reduced levels of XBP-1s transcripts associated with an increased DNA methylation of XBP-1 promoter [1]. We postulate that other epigenetic mechanisms, such as miRNAs, could coexist with promoter DNA hyper-methylation to regulate XBP-1s expression.

Objectives: Due to hsa-miR-513c-3p overexpression has been reported in SG from SS patients [2] and that XBP-1 is a predicted target of hsa-miR-513c-3p, the aim of this study was to evaluate whether mRNA levels of XBP-1s is modulated by hsa-miR-513c-3p and also if IFN-gamma modify the expression hsa-miR$513 c-3 p$ and XBP-1s.

Methods: SG biopsies from 16 SS-patients with low and high focus score and 5 controls were analyzed. hsa-miR-513c-3p levels were measured by Taqman miRNA assays, whereas XBP-1s mRNA levels were determined by qRT-PCR. Additionally, in vitro assays using IFN-gamma and chemically synthesized hsamiR-513c-3p mimics and inhibitors were performed to study its effect on XBP-1s expression.

Results: By Taqman assays we validated the overexpression of hsa-miR$513 c-3 p$ in SG from 8 SS patients with low $(p=0.03)$ and 8 SS-patients with high $(p=0.003)$ focus score, compared with SG from 5 controls. In the same samples, a decrease of XBP-1s transcript levels was observed in SG from SS-patients with low $(p=0.002)$ and high $(p=0.026)$ focus score. XBP1s transcript levels were negatively correlated with hsa-miR-513c-3p ( $r=-0.47, p=0.014)$, Ro $(r=-0.73$, $p=0.0009)$, ANA ( $r=-0.7, p=0.0033)$ and focus score $(r=-0.72, p=0.001)$. Stimulation of 3D-acini with $1 \mathrm{ng} / \mathrm{mL}$ IFN-gamma increase the hsa-miR-513c-3p levels $(p=0.014)$ and decrease the XBP-1s transcript levels $(p=0.027)$. A negative correlation was found between hsa-miR-513c-3p and XBP-1s transcript levels in $3 D$-acini stimulated with IFN-gamma $(r=-0.87, p=0.0001)$. The XBP-1s transcript levels were decreased in HSG cells transfected with hsa-miR-513c-3p mimic and increased in HSG cells transfected with the miRNA inhibitor.

Conclusion: IFN-gamma-induced upregulation of hsa-miR-513c-3p is consistent with the presence of STAT1-binding elements in its promoter region. Our findings suggest that the combined action of miRNAs and DNA methylation modulated by IFN-gamma could explain the altered expression of XBP-1s a key transcription factor involved in cellular proteostasis, affecting secretory function in LSG from SS-patients. Our results confirm previous correlations found between XBP-1s protein levels and clinical parameters of SS-patients, suggesting an association of XBP-1s with inflammation and impaired SG function.

References:

[1] D. Sepúlveda, et al, Rheumatology (Oxford), 2018;57:1021-32.

[2] I. Alevizos, et al,. Arthritis Rheum, 2011;63:535-44.

Acknowledgments : Fondecyt 1160015, Fondecyt Iniciación 11170049, Fondecyt Postdoctorado 3170023, CONICYT fellowship (PC, DJ).

Disclosure of Interests: : None declared

DOI: 10.1136/annrheumdis-2020-eular.2215

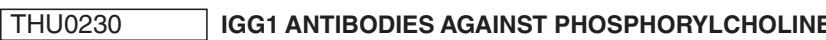 ARE NEGATIVELY ASSOCIATED WITH DISEASE ACTIVITY, DISEASE DAMAGE, CARDIOVASCULAR DISEASE AND ATHEROSCLEROSIS IN SLE: POTENTIAL UNDERLYING MECHANISMS.}

D. Thiagarajan ${ }^{1}$, R. Fiskesund ${ }^{1}$, J. Steen ${ }^{1}$, M. Rahman ${ }^{1}$, S. Lundström ${ }^{1}$, J. Frostegård ${ }^{1} .{ }^{1}$ Karolinska Institutet, Stockholm, Sweden

Background: Phosphorylcholine (PC) is an important component in cellular membranes and in lipoproteins that is exposed and recognized by the immune system, when cells undergo apoptosis or lipoproteins like LDL undergo oxidation. PC is also exposed in some microorganisms including nematodes and bacteria (non-self). We reported that IgM anti-PC is associated with protection in atherosclerosis, SLE, RA and other chronic inflammatory conditions. ${ }^{1}$ We also reported potential underlying protective mechanisms: 1 : increase in clearance of human dead cells, ${ }^{2} 2$ : inhibition of uptake of oxLDL in macrophages, 3: inhibition of cell death. ${ }^{1} 4$ : anti-inflammatory; 5: promotion of polarization of $T$ regulatory cells in SLE-patients T cells from a low level and also in plaque T cells. ${ }^{3}$ We generated in-house fully human IgG1 anti-PC clones for experimental studies to study anti-PC properties in humans. In contrast to mice, anti-PC are not germ-line encoded with a dominant clone. ${ }^{4}$

Objectives: We here study IgG1 and IgG2 anti-PC, with focus on atherosclerosis and SLE and properties of fully human IgG1 clones, in relation to SLE. Methods: We determined anti-PC by ELISA in 116 SLE-patients and 110 ageand sex-matched controls. For functional studies, we used three in-house generated, fully human monoclonal IgG1 anti-PC (A01, D05, E01). Apoptosis was induced in Jurkat T-cells and pre-incubated with A01, D05, E01 or isotype control IgG1 and effects on efferocytosis by human macrophages studied. Anti-PC peptide/protein characterization was determined using a proteomics de novo sequencing approach.

Results: IgG1 but not IgG2 anti-PC levels were higher among SLE patients $(p=0.02)$. IgG1 anti-PC was negatively associated with SLICC and SLEDAI (OR 2,978 Cl: $0.876-10.098$, OR: $5.108 \mathrm{Cl} 1.320 .067$ respectively) and negatively associated with CVD, atherosclerotic plaques and echolucent (potentially vulnerable plaques) but the association for the two former was not significant after controlling for confounders. D05 had maximum effect on macrophage efferocytosis efficiency, followed by A01 and E01. The monoclonal antibodies showed differential binding specificity to $\mathrm{PC}$ and $\mathrm{PC}$ associated neo-epitopes. Peptide analysis showed difference in the CDR3 region of the three anti-PC IgG1 clones which are crucial for recognition of $\mathrm{PC}$ on apoptotic cell surface and other neo-epitopes.

Conclusion: Anti-PC IgG1 is negatively associated with disease activity, and disease damage in SLE, but the negative association with CVD is also dependent on confounding risk factors. One potential underlying mechanism could be increased clearance of dead cells.

References:

[1] Frostegard J. Immunity, atherosclerosis and cardiovascular disease. BMC Med. 2013;11:117.

[2] Rahman M, Sing S, Golabkesh Z, Fiskesund R, Gustafsson T, Jogestrand T, Frostegard AG, Hafstrom I, Liu A and Frostegard J. IgM antibodies agains malondialdehyde and phosphorylcholine are together strong protection markers for atherosclerosis in systemic lupus erythematosus: Regulation and underlying mechanisms. Clin Immunol. 2016;166-167:27-37.

[3] Sun J, Lundstrom SL, Zhang B, Zubarev RA, Steuer J, Gillgren P, Rahman M, Ajeganova S, Liu A and Frostegard J. IgM antibodies against phosphorylcholine promote polarization of $\mathrm{T}$ regulatory cells from patients with 